\title{
Progresión de la gestión del patrocinio deportivo en España. ¿Siglo XXII?
}

\author{
Sandra Femenía Almerich \\ Universidad CEU Cardenal Herrera \\ sfemenia@uch.ceu.es
}

\begin{abstract}
Resumen:
Las crisis económicas agudizan el ingenio y es un periodo idóneo para apostar e innovar. Situaciones anteriores a la actual, han traído consigo el nacimiento de nuevos paradigmas en el ámbito de la comunicación, fruto de esa necesidad de cambio que conlleva un sector o mercado afectado por la recesión económica. El patrocinio, como técnica de la comunicación que es, lo ha vivido; y en el momento actual debe reinventarse y adaptarse, si no, morirá. En este trabajo se defiende que el patrocinio en el área del deporte debe gestionarse bajo una perspectiva relacional y/o de red, trabajando para forjar relaciones que permitan aprovechar sinergias entre los entes participantes.
\end{abstract}

Palabras clave: Gestores; patrocinio deportivo; enfoque relacional y/o de red.

\section{Progression of the management of sports sponsorship in Spain. 22th Century?}

\begin{abstract}
:
Economic crises deepen the wit and it is a suitable period to gamble and innovate. Prior to the current situations, they have brought about them new paradigms in the field of communication, fruit of this need for change that leads to a sector or market affected by the economic downturn. The sponsorship, as a technique of communication, has lived it; and at the present time it must reinvent itself and adapt and progress. This paper defends that the sponsorship in the sport area must be managed under a relational perspective and/or network, working to forge relationships that allow to the managers to take advantage of synergies between the participating entities.
\end{abstract}

Key words: Managers; sport sponsoring; relational approach and/ or network approach.

\section{Referencia normalizada:}

Femenía Almerich, S. (2014): Progresión de la gestión del patrocinio deportivo en España. ¿Siglo XXII?. Historia y Comunicación Social. Vol. 19. Núm. Especial Marzo. Págs. 575-585.

Sumario: Introducción: antecedentes generales. 2. Metodología. 3. Resultados. 4. Conclusiones. 5. Bibliografía. 6. Anexos. 7. Notas. 


\section{Introducción: Antecedentes generales}

Las crisis económicas siempre provocan cambios, concepto que altera la cotidianeidad, y que -en cierto modo- nos alienta a buscar alternativas, a innovar, a ser cautos, racionales, incluso, al mismo tiempo, atrevidos. En todas las épocas que España ha vivido una crisis, el sector de la comunicación se ha visto con la necesidad de realizar modificaciones. Hemos agudizado el ingenio dando como fruto nuevos paradigmas.

El patrocinio, como vehículo de la comunicación, también necesita una nueva adaptación, o, en el mejor de los casos, reinventarse. Ya antes de la crisis, el panorama de esta técnica, concretamente en el ámbito deportivo, no era muy alentador ${ }^{1}$. La escasa transparencia del sector, la intermediación de terceros, la falta de profesionalidad, la abundancia, etc. entre otros aspectos, obliga a que reflexionemos acerca de la imperante necesidad de progresar en el campo de la gestión del patrocinio en el deporte.

Los profesionales que se dedican a la gestión del patrocinio, bien desde el lado del patrocinado, bien desde la parte del patrocinador, bien sean independientes de ambos, necesitan saber cómo emplear estratégicamente el patrocinio desde un nuevo enfoque. Es momento de ello. La perspectiva relacional y/o de red permite una integración real de la marca en el evento y en el conjunto de firmas presentes, pudiendo maximizar su participación, incluso más allá del propio patrocinio.

Cornwell y Maignan (1998) hicieron hincapié en esta idea años antes, cuando consideraban que se fracasaba tanto por parte de académicos como por profesionales a la hora de entender cómo el patrocinio podría gestionarse. Un año después de que estas autoras expresaran esta aseveración tras el análisis de 80 artículos sobre el patrocinio, Hoek (1999) mostró que la gestión práctica del patrocinio necesitaba desarrollarse. Chadwick y Thwaites (2004) afirmaron que la literatura fracasó al examinar cómo se debía gestionar el proceso de patrocinio.

De los trabajos de Olkkonen, Tikkanen y Alajoutsijarvi (2000) y Olkkonen (2001) destacamos la manera en la que inciden sobre la importancia de que exista interacción entre varios miembros de la "red empresarial", además de destacar lo ventajoso de su participación en el proceso del patrocinio. También sugirieron que cuando se planificaba un patrocinio, las compañías necesitaban evaluar tanto el evento a apoyar y los recursos necesarios, como las correspondientes redes que pudieran surgir para, de este modo, vincular actividades y atar recursos con otros actores.

La mayoría de los estudios han investigado las relaciones que se establecen entre el patrocinador y el patrocinado como la clave para establecer un vínculo win-win, es decir, que ambos consigan sus objetivos con éxito².

La carencia de investigaciones para aclarar los contenidos de las relaciones de patrocinio, la falta de comprensión sobre cómo se producen las relaciones y las implicaciones teóricas y de gestión que ello puede tener, es una realidad y una demanda por parte de académicos que han trabajado este campo de estudio (Olkkonen, 2001). 
Aquellos estudiosos que han ido copando el campo de la gestión han puesto el punto de mira, principalmente, en las relaciones diádicas que se establecen, sin prestar atención a la gestión del patrocinio en el ámbito deportivo cuando colaboran en una misma actividad distintas marcas. El hecho de que varias firman patrocinen y colaboren en un mismo acontecimiento es lo habitual. De hecho, rara vez se encuentran casos donde una compañía actúa como patrocinadora única por voluntad de ésta, simplemente no sucede por cuestiones de carácter económico.

En un acontecimiento, todas las marcas (más o menos cantidad, según el tipo de actividad) están presentes compartiendo presencia, pero no entablan relación alguna ni interactúan. De este modo están desaprovechando la oportunidad de impactar como un todo en la mente del público, evitando posibles confusiones o que destaquen una o varias marcas sobre las demás.

Un gestor que trabaje el patrocinio deportivo siguiendo este nuevo enfoque, conseguirá que las marcas que patrocinan un mismo evento o actividad se beneficien de las sinergias que pueden surgir entre ellas, lo que a su vez será positivo para el evento por la unidad y la cohesión que transmitirá.

En este trabajo nos planteamos obtener un conocimiento más profundo de la gestión del patrocinio deportivo, con el fin de facilitar a los gestores las claves para operar, extrapolando las funciones que todo buen gestor debería desempeñar a nivel de patrocinio en un mercado competitivo como el actual y el futuro.

\section{Metodología empleada}

Como se ha apuntado al final del punto anterior, el planteamiento básico de esta investigación es recoger el estado de la situación de la gestión del patrocinio en España e identificar los aspectos clave de la gestión, que todo buen profesional debe conocer. Esto es, buscamos conocer cómo se efectúa la gestión cuando hay presencia de varias marcas en una misma actividad, tratando de comprender si su progresión hacia un nivel de desarrollo superior sería factible, posible y necesaria en el mercado español.

La concreción no se centra en la delimitación conceptual, si no que se trata de definir el problema entrando en contacto con él y sumergiéndonos mediante la lectura de lo publicado, así como conociendo el día a día de los profesionales que se encargan de su gestión.

Como dice Ruiz Olabuénaga, definir es: "situarse, orientarse, sumergirse, acercarse, contactar con el núcleo, el foco, el centro del mismo" (2003: 53).

Y continúa aclarando que esta operación incluye: "seleccionar un evento, una situación, un hecho, un comportamiento y delimitar el tiempo, el espacio, las personas, el contexto en donde uno se decide a investigar" Ibid. (p. 54). 
Por ello, hemos entrevistado a 25 gestores y consultores en patrocinio deportivo para que nos expliquen qué entienden por gestión del patrocinio deportivo con un enfoque relacional y/ o de red.

Para lograr nuestros objetivos hemos optado por realizar un estudio del sector mediante una investigación cualitativa de carácter descriptivo y exploratorio.

Para ello, en primer lugar, iniciamos el trabajo de investigación con la observación documental de la literatura existente reciente en torno a la gestión del patrocinio. Se trata de publicaciones que recogen las experiencias y aportaciones de gestores, asesores, directores de marketing, presidentes o gerentes de las compañías patrocinadoras. En fin, son aquellos máximos decisores en política de patrocinio, que independientemente del cargo y de su formación, aportan sus reflexiones en la bibliografía consultada, de la cual se extraen, de forma heurística ${ }^{3}$, las variables e indicadores que deben ser contrastados y verificados con los profesionales del patrocinio deportivo mediante la producción primaria de datos, extraídos de las entrevistas semiestructuradas en profundidad. Esta es la técnica empleada dentro de esta investigación de carácter descriptiva y exploratoria.

El trabajo continúa con la selección de la muestra y la puesta en contacto con ésta, para ajustar agendas y preparar los viajes de visita para efectuar las entrevistas en profundidad. Se ha entrevistado en dos fases a gestores y/o asesores consultores de patrocinio deportivo, para conocer desde un punto de vista neutro (ni desde la perspectiva del patrocinador, ni del patrocinado), cómo se está produciendo la gestión del patrocinio deportivo en la actualidad en España. Mediante el análisis de contenido de las entrevistas, se han extraído las claves de la investigación que nos ocupa. Hay que señalar que la incorporación de más aportaciones por más expertos no ha aportado novedad a los resultados, por lo que se decide detener la fase de entrevistas en el momento de detectar tal situación, por considerarse ya innecesario y no productivo.

El análisis del discurso de los actores del patrocinio nos ha permitido dibujar y analizar el estado de la situación de la gestión del patrocinio deportivo en España y conocer cómo afecta o afectaría al sector ensanchar la gestión tal y como se entiende y desarrolla en la actualidad, dotándola de un valor añadido al trabajarla desde una perspectiva relacional y/o de red.

Como resultado de esta investigación, se ofrecen diferentes conclusiones que justifican el objeto general de este trabajo. Y se construye un modelo de gestión del patrocinio múltiple con enfoque relacional y/o de red, plasmando las principales funciones que todo buen gestor de patrocinio debe desarrollar.

\section{Resultados}

Generalmente, todos los entrevistados coinciden en que el nivel de gestión del patrocinio deportivo en España está profesionalizado, especialmente en las empresas 
que actúan como patrocinadoras, cuya estructura interna les permite desde su planeación, búsqueda de patrocinios, ejecución y evaluación.

Los entrevistados han manifestado que las grandes empresas muestran tener una línea definida de patrocinio. Un reflejo del buen hacer es la integración de la activación como parte del patrocinio y de una gestión profesional. Mientras que las medianas y pequeñas corporaciones son vírgenes, carecen de enfoque y cuando patrocinan tienden a diversificar. No obstante, algunos manifiestan que a pesar de las completas estructuras y la planificación que tienen las grandes compañías en patrocinio, todavía siguen influyendo en las decisiones los gustos y preferencias del presidente, gerente o altos ejecutivos. Y en estos casos, el trabajo del gestor se encuentra detrás, tratando de justificar la pertinencia de ese patrocinio, y tratando de hacer encajar la imagen de marca del patrocinador con el patrocinado, para que sea creíble y convincente de cara al resto de públicos.

En cambio, hay patrocinados, competiciones, actividades, equipos o deportistas que aún demuestran carecer de know-how para emplear el patrocinio como una herramienta estratégica que permita a las empresas alcanzar sus fines comerciales y de comunicación.

Por eso, se repite en muchas entrevistas que España es todavía un país de contrastes (gestión profesional versus gestión amateur). Convive la existencia de todo un sector profesional (agencias especializadas, promotores, gestores, consultores, etc.) con acciones de patrocinio puntuales, organizadas tratando de obtener recursos y no anunciantes satisfechos, que no consideran necesario el apoyo de profesionales que les orienten sobre los pasos a seguir.

Para muchos de los entrevistados, la clave que ha conducido a una mayor profesionalización ha sido la labor de gestores y agencias especializadas independientes del patrocinado y del patrocinador que velan por los intereses de ambas partes por igual. Estos saben qué enfoque emplear para satisfacer las expectativas latentes del patrocinador, con el fin de garantizar su satisfacción y, por tanto, su fidelidad, además de haber incluido en su filosofía de trabajo la flexibilidad y la adaptación a las necesidades de patrocinador y patrocinado, sumado a un mercado más transparente donde todos los implicados asumen sus responsabilidades. Sin embargo, a través de sus intervenciones, muchos profesionales han relatado que esto no siempre ha sido así. El mercado del patrocinio deportivo ha evolucionado a partir de un mercado poco transparente, que funcionaba por amiguismo, manipulación y con intermediarios, "patrocinio a terceros", lo que condujo a que se enraizara en los anunciantes un sentimiento de incredulidad y suspicacia hacia la técnica. Ahora estamos ante un mercado perspicuo donde impera el estudio de la idoneidad de la operación, de la justificación y de la explicación continua, del ROI + el ROO para demostrar la efectividad de su impacto.

La situación económica en la que se encuentra España ha contribuido tanto para bien, como para mal. El aspecto positivo ha sido el aumento de la profesionalización, puesta en duda por la abundancia (económica) y la mala gestión (por parte de las 
empresas y de los patrocinados/gestores/agencias). Es decir, años anteriores, cuando las empresas han contado con suficiente capital, han invertido sin cuestionarse estratégicamente la idoneidad de ello. Al mismo tiempo, los gestores o agencias han desarrollado una gestión poco profesional, poco trasparente y en ocasiones, fraudulenta. En la actualidad se están agudizando los sentidos de los gestores, de los anunciantes y de los patrocinados para poder hacer frente a los cambios. Hay por tanto un aumento del know-how, de la conciencia de las marcas por obtener resultados de cada céntimo invertido y de la necesidad de materializarlo. La consecuencia negativa proviene del receso de inversión por parte de aquellos anunciantes, "patrocinadores incrédulos", que frenan sus patrocinios o tienden a la diversificación, lo que conlleva la desaparición de patrocinados (eventos pequeños y deportes minoritarios sin estructura y sin conocimiento para rentabilizar la inversión, principalmente). $\mathrm{Y}$ al haber una menor inversión por parte de las empresas privadas, se recurre a las instituciones públicas para que se hagan cargo e impidan su desaparición.

Pero no es posible sistematizar lo dicho, ya que por parte de ciertos sectores (entidades financieras, por ejemplo) y en modalidades deportivas concretas (competiciones de carácter mundial, por ejemplo), se está aumentando la inversión.

Un aspecto pernicioso para algunos de los entrevistados, es la proliferación de patrocinios con carácter social y de utilidad pública que permiten a las empresas beneficiarse de ventajas fiscales. No es que se muestren en contra de ellos, si no que debido a la crisis, se ha extendido la búsqueda por parte de las empresas de este tipo de actividades, en detrimento de las demás.

Volviendo al inicio de este apartado, según los entrevistados, la gestión del patrocinio deportivo en España se presenta mudable, pues dependerá de quién la efectúe (agente/ gestor de la marca o patrocinador; agente/ gestor del patrocinado; agente/ gestor intermediario ajeno al patrocinador y al patrocinado), del evento o actividad, de la modalidad deportiva y de la/s empresa/s implicada/s, como se recoge a continuación (tabla 1):

Tabla 1: Factores que influirán en la Gestión del Patrocinio Deportivo en España.

\begin{tabular}{|l|l|l|l|}
\hline \multicolumn{1}{|c|}{ QUIÉN } & SOBRE QUÉ & DÓNDE & PATROCINADORES \\
\cline { 1 - 4 } Agente o Gestor del Patrocinador & $\begin{array}{l}\text { El Evento o } \\
\text { Actividad/ }\end{array}$ & $\begin{array}{l}\text { Modalidad } \\
\text { deportiva }\end{array}$ & Empresas implicadas \\
\cline { 1 - 3 } Agente o Gestor del Patrocinado & Patrocinado & & \\
\cline { 1 - 3 } $\begin{array}{l}\text { Agente o Gestor Intermediario ajeno } \\
\text { al patrocinador y al patrocinado }\end{array}$ & & & \\
\hline
\end{tabular}

\section{Conclusiones}

La cantidad de patrocinios múltiples en España es muy superior a los eventos con un patrocinio único o single, por la necesidad de tener que diversificar riesgos -por parte del patrocinado y/o su gestor-, así como para captar los recursos necesarios que 
se requieren para desarrollar su actividad. Mientras que el gestor del patrocinador o, éste en su defecto, comparte patrocinios por la dificultad de respaldarlo (económica $\mathrm{y} / \mathrm{o}$ en especie) en solitario.

La situación se caracteriza por contar con un alto nivel de profesionalización de la gestión del patrocinio deportivo, lo que da oportunidad a la implantación de una gestión de nivel superior donde el patrocinio siga un enfoque relacional y/o de red de relaciones.

Aquellas empresas con experiencia en patrocinio son las más proclives a poner en marcha un patrocinio relacional o de red, o de dejarse guiar (por gestor o agencia) para formar parte de él. Ahora bien, dependerá de factores como: los objetivos, los posibles socios, la flexibilidad del gestor, el presupuesto, el público al que se dirigen, etc. el que lo aplique con ese enfoque o no. Las grandes compañías suelen planteárselo desde una perspectiva estratégica, o al menos tratan de justificarlo como tal, aunque es cierto que las medianas y pequeñas empresas también lo protagonizan, pero por una cuestión de oportunismo causal (compartir costes, know-how e imagen).

Las empresas (patrocinadores) han tenido más background en la gestión del patrocinio que los patrocinados. Son las pioneras, activa o reactivamente en aceptar la configuración del patrocinio desde la perspectiva relacional y/o de red, aunque también las hay que sólo se mueven por la relación y trasferencia de valores que exista entre ellas y el evento y no con el resto de patrocinadores. Mientras que los patrocinados suelen dar aquellos pasos que el patrocinador o potencial patrocinador les marcan, ya que continúa siendo el patrocinador el que tiene el control y el dominio sobre la relación patrocinador-patrocinado y entre patrocinadores.

Distinto es en el caso de los gestores o agencias especializadas en patrocinio, cuyo negocio se centra en el patrocinio y en la satisfacción a largo plazo de patrocinados y patrocinadores, puesto que si quieren distinguir el producto que comercializan, éste deberá aportar valor a las partes, y el enfoque relacional y/o de red es la clave, ya no sólo para diferenciar aquello que comercializan, sino para generar complacencia, eficacia, eficiencia, compromiso y fidelidad a largo plazo entre los entes implicados.

Por tanto, concluimos que encontramos una parte del mercado español del patrocinio deportivo donde impera la idoneidad de la operación, la justificación, la explicación continua, el ROO y el ROI, el aumento del know-how, de la conciencia de las marcas por obtener resultados de cada céntimo invertido y la necesidad de materializarlo. Este es el contexto necesario para desarrollar un patrocinio relacional y/o de red. Y, por otra, convive con una gestión menos profesional, desarrollada por anunciantes que cuestionan el patrocinio y sus efectos y de gestores o agencias poco preparadas. Ello se combina con la recesión económica, que ha provocado 4 situaciones generales: 1) abandono del patrocinio por parte de empresas privadas; 2) reducción de la participación en el patrocinio por parte de empresas privadas y/o públicas; 3 ) incorporación con más protagonismo de las instituciones públicas ejerciendo el rol de patrocinadoras, que puede ralentizar la incorporación de la gestión relacional o de 
red en el patrocinio en nuestro país; 4) mayor esfuerzo en racionalizar la inversión y en innovar por parte, especialmente de las marcas.

Así pues, como consecuencia de un marco de patrocinio deportivo en España mudable, dependerá de quién efectúe la gestión, de la propiedad deportiva, de la modalidad deportiva y del conjunto de empresas implicadas el que se pueda explotar o no el patrocinio con una visión relacional y/o de red.

Para finalizar, recogemos las principales funciones que un gestor profesional de patrocinio en el ámbito deportivo debería desempeñar, aclarando qué entienden la muestra entrevistada. Son las siguientes:

- Transparente: el gestor no debe ocultar información a los patrocinadores. Debe ser claro, profesional, compartir la información precisa y justa para que los patrocinadores confien en su gestión, es decir, que un gestor transparente no tiene que ir desvelando las estrategias y objetivos de todos los anunciantes involucrados, si no respetar la privacidad de cada firma y no tratar de engañarla en ninguna parte del proceso.

- Ejecutor: llamamos así a aquel gestor que se encarga de explotar el patrocinio junto al patrocinador y/o su agencia de comunicación o especializada en patrocinio.

- Comunicador: el gestor debe tener la habilidad para sintonizar e interactuar continuamente con el patrocinado y con cada marca para que de esta relación puedan surgir mejores resultados. De sus conversaciones pueden surgir proyectos de gran interés que enriquezcan su vínculo y aporten al patrocinio un valor añadido.

- Informador: el gestor tiene que transmitir a las marcas involucradas en el patrocinio los avances y los progresos de la gestión, como por ejemplo: qué nuevas incorporaciones (de patrocinadoras) se han producido, a qué sectores pertenecen, resultados de ediciones anteriores, iniciativas con éxito de ediciones anteriores, etc.

- Comercial: es aquel gestor que además de estudiar cuáles son las necesidades del patrocinado y de los potenciales patrocinadores, adaptar el proyecto que representa a éstas y estructurar el programa de patrocinio, se encarga de venderlo a los anunciantes que ha considerado idóneos para que actúen como patrocinadores.

- Educador: es aquel gestor que mientras desempeña su labor, inicia un rol lúdico formativo de cara al patrocinado y/o al potencial patrocinador, enseñándoles las ventajas e inconvenientes de elaborar cierta gestión del patrocinio.

- Asesor: asesorar debería ser una labor inherente al gestor, el cual, debería guiar durante todo el proceso al patrocinador. Su fin principal es que éste obtuviese el máximo nivel de satisfacción con respecto al patrocinio, por lo que aprovecharse de la experiencia del gestor puede ayudar a su consecución. El problema al que se enfrenta el gestor en el mercado español es la falta de conciencia por parte de los anunciantes con respecto a esta acción, pues la tendencia es no dejarse asesorar, adquirir el programa de patrocinio y actuar independientemente del gestor por la falta de confianza que despierta en ellos. 
- Colaborador: gestor y patrocinadores trabajarían como compañeros durante todo el proceso, ayudándose para la obtención del beneficio mutuo.

- Negociador: el gestor tiene que estructurar la propuesta de patrocinio en base a unos criterios y ofrecérsela a cada potencial patrocinador tratando de que culmine con la venta completa del patrocinio. Tendrá que convenir con cada marca, analizando su situación y ofreciéndole aquello más acorde para ésta, adaptándose, siendo flexible.

- Flexible: el gestor de hoy en día tiene adaptarse a la situación del mercado, de las características del patrocinado y a las del patrocinador, para que el proyecto que representa sea susceptible de cambios o variaciones según las circunstancias, pero sin perder la identidad originaria de éste.

- Controlador: forma parte de las funciones de muchos gestores el tener que orientar, regular y vigilar para que ninguna marca -especialmente de entre las situadas en la misma categoría- sobresalga sobre el resto, y menos aún, sobre las situadas en categorías superiores.

- Proactivo: el gestor que actúa reactivo plantea actividades, acciones, proyectos, etc. adelantándose a las necesidades del patrocinador. Se caracteriza por tener él la iniciativa y proponerla a la marca o marcas afectadas por su idea.

- Reactivo: un gestor reactivo es el que responde ante las sugerencias de uno o más patrocinadores. La iniciativa es continuada por él, no iniciada por él.

- Polivante: es el gestor que trabaja para el patrocinado, es decir, efectúa su gestión del patrocinio, pero mira por el beneficio del patrocinador también.

- Profesional: un gestor profesional es aquel que no sólo se encarga de vender la propuesta, si no que se implica e integra en ella para que todas las partes (él mismo, el patrocinado y el patrocinador) obtengan un beneficio a largo plazo.

- Estratega: el gestor se encarga de definir una estrategia para comercializar el patrocinio, pero a su vez, se preocupa de integrar la estrategia de cada marca en el patrocinio propuesto.

- Receptivo: esta actitud viene de la mano, en numerosas ocasiones, de la flexibilidad, es decir, que el gestor suele tener una mayor disposición a esperar, pero al mismo tiempo a hacer frente, con ánimo de facilitar aquello que nece$\operatorname{sita}(n)$ el patrocinador y/o el patrocinado.

- Analista: el análisis forma parte la labor de un estratega, por lo que tendría que ser inherente a la función del gestor profesional. Estudio y análisis del patrocinado, de sus características y de sus necesidades, análisis del mercado para adaptarse a él sin efectuar una gestión miope, análisis del potencial patrocinador, análisis de las convergencias, análisis de las sinergias, etc.

- Facilitador o Abastecedor: siguiendo la acepción que la RAE efectúa de este adjetivo, podríamos decir que ser facilitador es sinónimo de ser asesor, ya que 
se entiende como tal a aquella persona que se desempeña como instructor $u$ orientador, lo que podría llegar a encajar en la definición de asesor, pero vamos más allá con esta función, pues con facilitador el gestor trata de instruir y de conseguir aquello que el patrocinado $\mathrm{y} / \mathrm{o}$ el patrocinador precisan.

- Reputado: el gestor tiene que ser reconocido, dentro del sector, como experto y profesional en su profesión.

- Observador: el gestor tiene que vigilar el patrocinio que se desempeña en otros niveles que pueda llegar a afectar a los patrocinadores del nivel que él maneja.

\section{Bibliografía}

CHADWICK, S., THWAITES, D. (2005). "Managing Sport Sponsorship Programs: Lessons from a Critical Assessment of English Soccer". En Journal of Advertising Research, p. 328-338.

CORNWELL, B.T.; MAIGNAN, I. (1998). "An international review of sponsorship research". En Journal of Advertising, no 27, (1), p. 1-30.

HOEK, J. (1999). "Sponsorship: An evaluation of management assumptions and practices". En Marketing Bulletin, $\mathrm{n}^{\mathrm{o}}$ 10, p. 1-10.

OLKKONEN, R.; TIKKANEN, H.; ALAJOUTSIJARVI, K. (2000). "Sponsorships as relationships and networks: implications for research". En Corporate communications, $\mathrm{n}^{\mathrm{o}} 5$, (1), p. 12-21.

OLKKONEN, R. (2001). "Case study: the network approach to international sport sponsorship arrangement". En Journal of business and industrial marketing, $\mathrm{n}^{\mathrm{o}}$ 16, (4), p. 309-329.

RUIZ OLABUÉNAGA, J. I. (2003). Metodología de la investigación cualitativa. Bilbao: Universidad de Deusto. $3^{\text {a }}$ Edic.

\section{Notas}

1 Véase volumen de inversión en Patrocinio Deportivo en www.infoadex.es

2 Véase Chadwick, 2004; Mason (1999) Hoek (1998) que identificaron el patrocinio como una forma de colaboración estratégica.

3 La hermenéutica emplea un proceso centrado en la interpretación más subjetiva para poder entender mejor la realidad. Un hermeneuta interpreta sucesos inmediatos por medio de experiencias anteriores y de cualquier elemento que le permita entender la situación de estudio. Por contra, como explica Ruiz Olabuénaga, el Positivismo se basa en el análisis estadístico de datos recogidos por medios de estudios y experimentos de carácter descriptivos y comparativos. 


\section{La autora}

Sandra Femenía Almerich es Licenciada en Publicidad y RR.PP. y Doctora en Comunicación y Profesora Universitaria en la Facultad de Humanidades y Ciencias de la Comunicación de la Universidad CEU Cardenal Herrera. 